\title{
Why people failed to adhere to COVID-19 preventive behaviors? Perspectives from an integrated behavior change model
}

\author{
Derwin K. C. Chan PhD ${ }^{1,2,3}$ (1), Chun-Qing Zhang PhD ${ }^{2,4}$ and Karin Weman-Josefsson PhD 5 \\ ${ }^{1}$ Faculty of Education and Human Development, The Education University of Hong Kong, Hong Kong SAR, China, ${ }^{2}$ School of Psychology, Curtin University, Perth, \\ Australia, ${ }^{3}$ School of Public Health, The University of Hong Kong, Hong Kong SAR, China, ${ }^{4}$ Department of Sport and Physical Education, Hong Kong Baptist \\ University, Hong Kong SAR, China and ${ }^{5}$ Center for Research on Welfare, Health and Sport, Halmstad University, Halmstad, Sweden
}

To the Editor-Many preventive behaviors such as the practice of hand, personal, and respiratory hygiene; maintaining social distance (eg, staying home); and cleaning and disinfection are recommended for the prevention of the new coronavirus (COVID-19). However, a growing number of reports have revealed

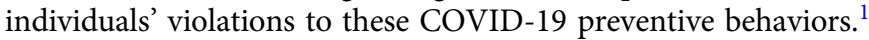
These violations might endanger the community by increasing the risk of an outbreak of COVID-19. The uptake of and adherence to health behaviors, including behaviors related to the prevention of infectious diseases (eg, COVID-19), are likely highly dependent on individuals' motivation, intention, and other decision-making factors. ${ }^{2}$ We aim to apply an integrated behavior change model of health psychology to explain why individuals fail to comply and adhere to these behaviors.

\section{The integrated model}

The integrated model of self-determination theory ${ }^{3}$ and the theory of planned behavior ${ }^{4}$ is a behavior change model that utilizes the concepts of 2 widely used psychological theories. The integrated model outlines the processes by which psychological need support, and motivations directly and indirectly link to the social cognition beliefs, intention, and behavior (Fig. 1). ${ }^{5}$ In the integrated model, ${ }^{5}$ when social environments are supportive to individuals' basic psychological needs of autonomy, competence, and relatedness, individuals are more likely to endorse autonomous motivation (ie, acting for inherent interest, satisfaction, personal goals, and values) than controlled motivation (ie, acting due to external contingencies, internal pressure, or sense of ego). They are also more likely to have more favorable social cognition beliefs (ie, attitude, subjective norm, and perceived behavioral control) and intentions and to demonstrate behavioral adherence in health behaviors. The psychological pathways illustrated in the integrated model have been supported by evidence from various health contexts and cultures, ${ }^{5,6}$ including preventing $\mathrm{H} 1 \mathrm{~N} 1$ transmission during a pandemic. ${ }^{2}$ We believe that the integrated model can explain why some people have failed to adhere to the recommended behaviors for COVID-19 prevention.

Author for correspondence: Derwin K. C. Chan, E-mail: derwin@eduhk.hk

Cite this article: Chan DKC, Zhang C-Q, and Weman-Josefsson K. (2021). Why people failed to adhere to COVID-19 preventive behaviors? Perspectives from an integrated behavior change model. Infection Control \& Hospital Epidemiology, 42: 375-376, https://doi.org/10.1017/ice.2020.245

\section{Why people do or do not adhere to COVID-19 prevention recommendations}

\section{Law enforcement}

A number of countries have set up legislation regarding social distance measures (eg, stay-home restriction), quarantine, and lockdown/travel ban. These legislative actions are classic examples of external factors that foster the development of controlled motivation. According to the integrated model, ${ }^{5}$ individuals who are driven by controlled motivation (ie, acting due to external contingencies, internal pressure, or sense of ego) may adhere to the advisory behavior as soon as the external factors (eg, contingencies of following COVID-19 preventive behaviors or not) are present, but they are more vulnerable to nonadherence in the long term than those who hold autonomous motivation (ie, acting for inherent interest, satisfaction, personal goals, and values) for the action. Individuals driven by controlled motivation alone might consider violations of health legislations when they perceive that the risks of getting caught or negative health consequences are low. ${ }^{7}$ At present, the enforcement of some new COVID-19 prevention legislations (eg, social distancing measures) could be extremely challenging when the surveillance involves a large geographical area or population. Governments or public health organizations should consider noncoercive strategies that are aligned with basic psychological needs to foster individuals' autonomous motivation of COVID-19 prevention.

\section{Social and environmental factors}

In addition to law enforcement, other social situations and environmental factors are supportive or detrimental to the motivational and social cognition factors affecting COVID-19 prevention. In support to the psychological factors in the integrated model $^{5}$, there are social situations or personal beliefs that facilitate autonomous motivation (eg, "preventing COVID-19 is what I want to do because I am responsible for my own health"), attitude (eg, accessible online information about the values of COVID-19 prevention), subjective norms (eg, family or friends who are following the COVID-19 preventive strategies say I should do the same), and perceived behavioral control (eg, training resources that make it easier for me to correctly apply COVID-19 preventive behavior such as hand hygiene). In contrast, some social circumstances are detrimental to motivational and social cognition factors. For instance, advice on the necessity of wearing face masks in community settings has been inconsistent across different nations and health organizations, ${ }^{8}$ which might discourage individual autonomous motivations (eg, "Do I really want to prevent COVID-19 in this way?") and attitudes 


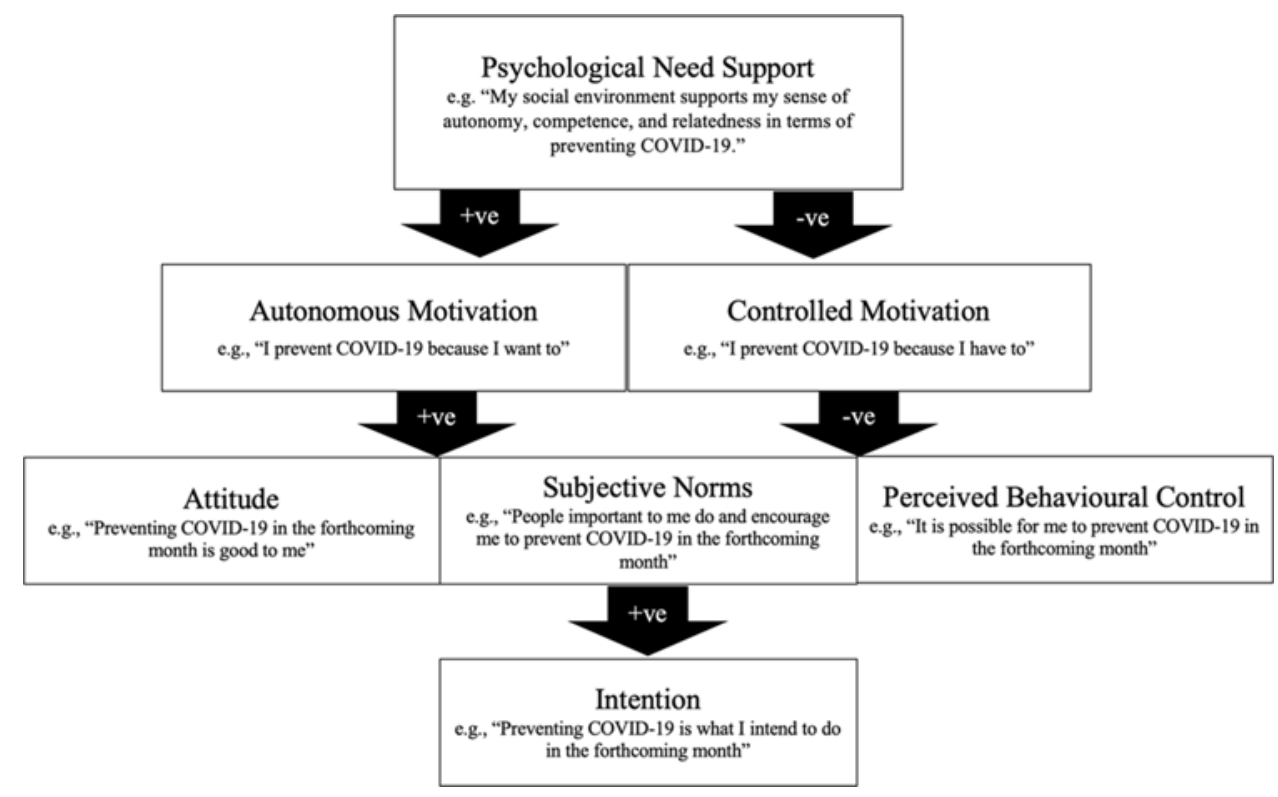

Fig. 1. The integrated model of self-determination theory and the theory of planned behavior.

(eg, "Are there any points to wearing a face mask for the prevention of COVID-19?"). The shortage of personal protective equipment (PPE) $)^{9}$ might impair an individual's sense of competence and perceived behavioral control (eg, "lack of PPE has made the prevention of COVID-19 challenging and uncontrollable"). Discrimination toward, alienation of, and labeling of individuals who wear face masks in public areas ${ }^{10}$ or social groups that encourage the ignorance of social distance measures ${ }^{1}$ might undermine an individual's relatedness and subjective norms in the context of COVID-19 prevention. Governments and health organizations should be aware of these factors and should implement policies and social strategies that facilitate the motivational and social cognition factors affecting COVID-19 prevention.

In conclusion, the integrated model of self-determination theory and the theory of planned behavior explains why some individuals fail to adhere to the preventive behaviors of COVID-19. We hope our discussion may raise the awareness of governing bodies and public health sectors regarding the importance of considering individuals' motivation and social cognition beliefs when implementing COVID-19 preventivon measures in the community.

\section{Acknowledgments. None.}

Financial support. No financial support was provided relevant to this article.

Conflicts of interest. All authors report no conflicts of interest relevant to this article.

\section{References}

1. Fears 'lockdown parties' will increase global spread of coronavirus. 2020. The Guardian website. https://www.theguardian.com/world/2020/mar/ 18/fears-lockdown-parties-will-increase-global-spread-of-coronavirus. Accessed March 18, 2020.

2. Chan DKC, Yang SX, Mullan B, et al. Preventing the spread of H1N1 influenza infection during a pandemic: autonomy-supportive advice versus controlling instruction. J Behav Med 2015;38:416-426.

3. Deci EL, Ryan RM. Intrinsic Motivation and Self-Determination in Human Behavior. New York: Plenum; 1985.

4. Ajzen I. From intentions to actions: a theory of planned behavior. In: Kuhl J, Beckmann J, eds. From Intentions to Actions: A Theory of Planned Behavior. Berlin: Springer; 1985. Pp. 11-39.

5. Hagger MS, Chatzisarantis NLD. Integrating the theory of planned behaviour and self-determination theory in health behaviour: a metaanalysis. Br J Health Psychol 2009;14:275-302.

6. Hagger MS, Chatzisarantis NL. The trans-contextual model of autonomous motivation in education: conceptual and empirical issues and meta-analysis. Rev Educ Res 2016;86:360-407.

7. Chan DKC, Dimmock JA, Donovan RJ, Hardcastle S, Lentillon-Kaestner V, Hagger MS. Self-determined motivation in sport predicts anti-doping motivation and intention: a perspective from the trans-contextual model. J Sci Med Sport 2015;18:315-322.

8. Feng S, Shen C, Xia N, Song W, Fan M, Cowling BJ. Rational use of face masks in the COVID-19 pandemic. Lancet Respir Med 2020;8: 434-436.

9. Ranney ML, Griffeth V, Jha AK. Critical supply shortages- the need for ventilators and personal protective equipment during the Covid-19 pandemic. N Engl J Med 2020. 382:e41. doi: 10.1056/NEJMp2006141.

10. Leung $\mathrm{H}$. Why wearing a face mask is encouraged in Asia, but shunned in the U.S. 2020. Time website. https://time.com/5799964/coronavirus-facemask-asia-us/. Accessed April 4, 2020. 